\title{
Assessment of blood flow velocities and venous pressures using a dual-sensor guidewire in symptomatic dural sinus stenoses
}

\author{
Stéphanie Lenck, MD, ${ }^{1}$ Fabrice Vallée, MD, ${ }^{2}$ Vittorio Civelli, MD, ${ }^{1}$ Jean-Pierre Saint-Maurice, MD, ${ }^{1}$ \\ Patrick Nicholson, MD, ${ }^{3}$ Alex Hong, MD, ${ }^{2}$ and Emmanuel Houdart, MD ${ }^{1}$
} Departments of ${ }^{1}$ Neuroradiology and ${ }^{2}$ Anesthesiology and Critical Care, Hôpital Lariboisière, Paris, France; and ${ }^{3}$ Department of
Neuroradiology, Toronto Western Hospital, University Health Network, Toronto, Ontario, Canada

\begin{abstract}
Lateral venous sinus stenoses have been associated with idiopathic intracranial hypertension and venous pulsatile tinnitus. Venous pressure measurement is traditionally performed to assess the indications for stenting in patients with idiopathic intracranial hypertension. However, its reliability has recently been questioned by many authors. The dualsensor guidewire was first developed for advanced physiological assessment of fractional and coronary flow reserves in coronary artery stenoses. It allows measurement of both venous pressure and blood flow velocities. The authors used this device in 14 consecutively treated patients to explore for symptomatic lateral sinus stenosis. They found that venous blood flow was significantly accelerated inside the stenotic lesion. This acceleration, as well as the pulsatile tinnitus, resolved in all patients following stent placement. According to the authors' results, this guidewire can be helpful for establishing an indication for stenting in patients with pulsatile tinnitus and idiopathic intracranial hypertension.
\end{abstract}

https://thejns.org/doi/abs/10.3171/2017.12.JNS172364

KEYWORDS idiopathic intracranial hypertension; pulsatile tinnitus; venous sinus stenoses; dual-sensor guidewire; diagnostic technique; vascular disorders

I NTRACRANIAL lateral venous sinus (LS) stenoses have been associated with idiopathic intracranial hypertension (IIH) $)^{12}$ and venous pulsatile tinnitus (PT). ${ }^{2,6}$ The measurement of the venous transstenotic pressure gradient (TSG) is traditionally performed in patients with IIH to assess the indications for stenting. ${ }^{12}$ However, both the reliability of this technique and the value of the threshold TSG have been questioned by many authors. ${ }^{4,7}$ The clinical assessment of PT may be challenging in patients without IIH, and the discovery of LS stenoses can be incidental. ${ }^{5}$ It can be therefore difficult to ascertain the role that these stenoses play in patients with PT. There is a clear need for a more precise diagnostic tool to help determine reliable hemodynamic criteria, which will help better predict which patients with symptomatic LS stenoses will benefit from stenting. A dual-sensor wire is commonly used in cardiology to measure both pressures and velocity across an atherosclerotic lesion. This physiological approach has proven more beneficial in the selection of therapeutic targets for coronary revascularization compared with measurement of pressures alone. ${ }^{9}$

The purpose of the present study was to evaluate the hemodynamic consequences of symptomatic dural-sinus stenoses in terms of venous blood flow velocities (BFVs) and venous pressures in a prospective series of 14 patients in whom exploration was performed using a dual-sensor pressure and Doppler velocity guidewire.

\section{Methods}

After receiving institutional review board approval, we collected the clinical, radiological, and hemodynamic data of patients in whom exploration was performed using a dual-sensor guidewire (ComboWire, Volcano Corporation [Philips]) for a disabling venous PT, with or without IIH, associated with an ipsilateral LS stenosis confirmed on imaging. Written informed consent was obtained before the procedure for all patients according to local institutional protocol. All patients underwent an otoscopic examination, MRI with MR angiography and MR venography, and bone CT scanning to exclude other causes of PT.

Using local anesthesia, the diagnostic procedures were performed via a 5-F venous femoral sheath. The stent placement and the poststenting hemodynamic measure-

ABBREVIATIONS BFV = blood flow velocity; IIH = idiopathic intracranial hypertension; $L S=$ lateral sinus; $\mathrm{PT}=$ pulsatile tinnitus; TSG = transstenotic pressure gradient. SUBMITTED September 21, 2017. ACCEPTED December 19, 2017. 
TABLE 1. Hemodynamic values measured with the dual-sensor ComboWire guidewire and the manometric measurement

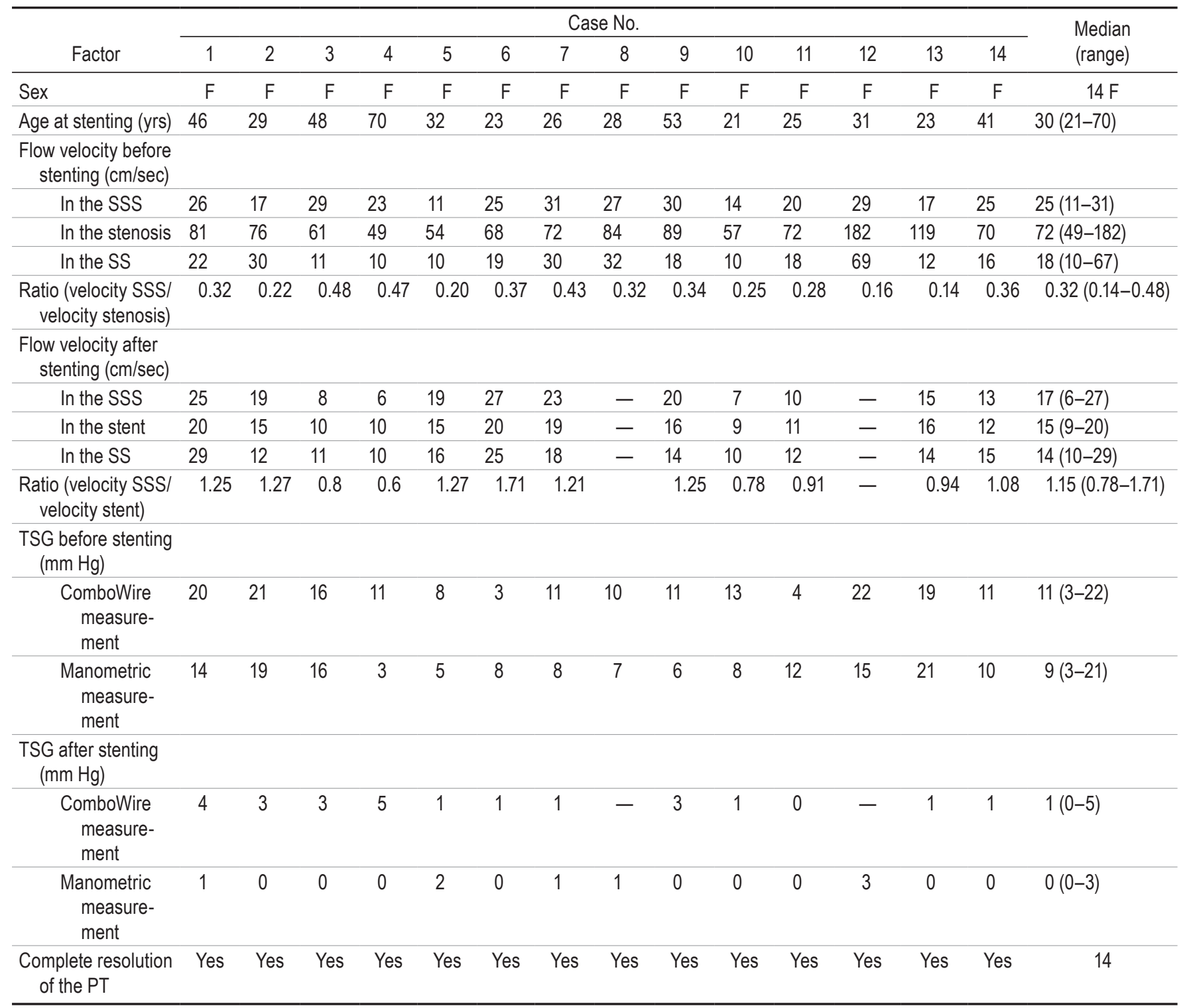

SS = sigmoid sinus; SSS = superior sagittal sinus; $-=$ not measured.

ments were performed with the patient under general anesthesia, via an 8-F jugular sheath. A Traxcess-14 microwire (MicroVention) was used to navigate a 21-inch Prowler Select Plus microcatheter to the origin of the superior sagittal sinus. Venous pressure and the BFV were measured at the origin of the superior sagittal sinus, at the site of maximal sinus stenosis, or in the stent and in the sigmoid sinus before and after stent placement. A 0.014-inch dual-sensor pressure and Doppler velocity ComboWire was used in combination with ComboMap matching hardware to measure the venous pressures and the BFV. All pressure and velocity signals were recorded at $200 \mathrm{~Hz}$. In addition, the venous pressures were measured using the microcatheter through an invasive arterial transducer (manometric measurement) and were compared with the venous pressures measured with the dual-sensor guidewire.

\section{Statistical Analysis}

Statistical analyses were performed with Stata software (StataCorp). Quantitative variables are expressed in median. Statistical analyses were performed using a Wilcoxon matched-pairs signed-rank test for continuous variables. A $\mathrm{p}$ value $<0.05$ was considered significant.

\section{Results}

Fourteen patients (all female) were included in our study: 6 with an isolated venous PT and 8 with venous PT associated with IIH. The median age at symptom onset was 26.5 years (range 12-69 years), and the median age at time of stenting was 30.0 years (range $21-70$ years). The median BMI was $26.8 \mathrm{~kg} / \mathrm{m}^{2}$ (range $21-38 \mathrm{~kg} / \mathrm{m}^{2}$ ). The PT immediately resolved following stent placement in all patients. 


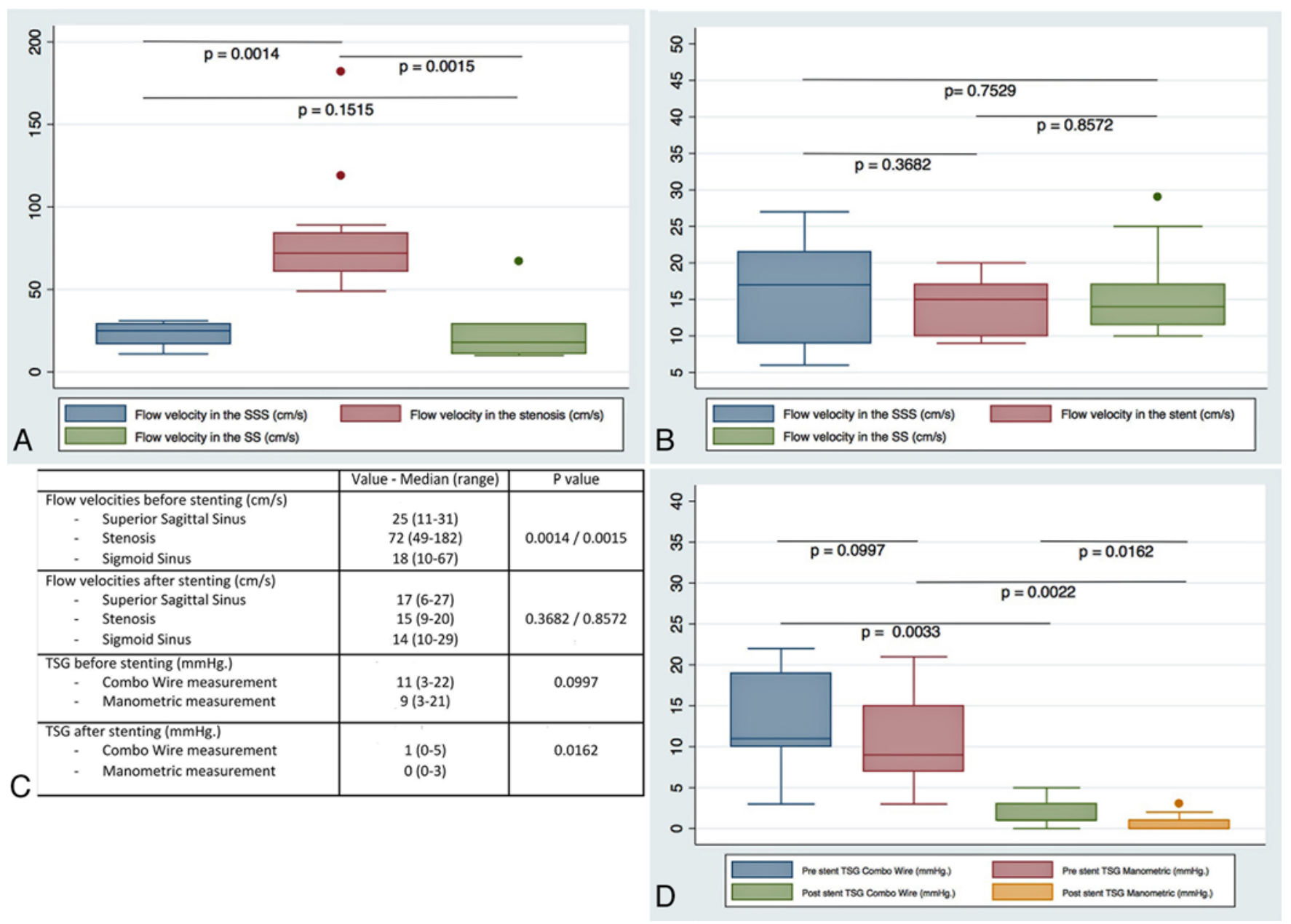

FIG. 1. Hemodynamic assessment of patients with intracranial venous sinus stenoses using manometric and dual-sensor guidewire (ComboWire) measurements. A: BFV measured with the ComboWire before stenting showing a significant acceleration of the flow inside the stenosis. B: BFV measured with the ComboWire after stenting showing the resolution of the BFV acceleration. C: Summary table of hemodynamic data. D: Venous TSG before and after stent placement with the ComboWire and the manometric measurements. SS = sigmoid sinus; SSS = superior sagittal sinus. Figure is available in color online only.

The hemodynamic data are summarized in Table 1 and Fig. 1. Before stent placement, the BFV was significantly increased at the site of the stenosis $(72 \mathrm{~cm} / \mathrm{sec}$ [range $49-182 \mathrm{~cm} / \mathrm{sec}])$ compared to the BFV above $(25 \mathrm{~cm} / \mathrm{sec}$ [range $11-31 \mathrm{~cm} / \mathrm{sec}] ; \mathrm{p}=0.0014)$ and below $(18 \mathrm{~cm} / \mathrm{sec}$ [range $10-67 \mathrm{~cm} / \mathrm{sec}$ ]; $\mathrm{p}=0.0015$ ) the stenosis. Following stent placement, this differential in BFV decreased; the poststenting BFV was not statistically different when measured in the stent $(15 \mathrm{~cm} / \mathrm{sec}$ [range 9-20 cm/sec]) compared to above $(17 \mathrm{~cm} / \mathrm{sec}$ [range 6-27 $\mathrm{cm} / \mathrm{sec}$; $\mathrm{p}=$ $0.3682)$ and below $(14 \mathrm{~cm} / \mathrm{sec}$ [range $10-29 \mathrm{~cm} / \mathrm{sec}$ ]; $\mathrm{p}=$ 0.8572 ) the stent. A ratio (velocity in the superior sagittal sinus/velocity in the stenosis) of less than 0.5 was observed in all patients before stenting, suggesting a significant acceleration of the flow inside the stenosis, and this ratio was always $>0.5$ after stent placement. The TSG also significantly decreased after stent placement when measured with both the invasive transducer ( 9 vs $0 \mathrm{~mm} \mathrm{Hg}$, $\mathrm{p}=0.0033$ ) and the dual-sensor guidewire (11 vs $1, \mathrm{p}=$ 0.0022). Before stenting, there was no significant differ- ence in the TSG when measured with the invasive transducer and the dual-sensor guidewire (11 vs $9 \mathrm{~mm} \mathrm{Hg}$, p $=0.0997)$. The poststenting TSG was statistically higher when measured with the dual-sensor guidewire compared with the invasive transducer ( $1 \mathrm{vs} 0 \mathrm{~mm} \mathrm{Hg}, \mathrm{p}=0.0162$ ).

\section{Discussion}

The TSG is traditionally used to assess the indication for LS stenting in patients with IIH..$^{11}$ It is usually measured using an invasive arterial transducer connected to a microcatheter. However, the reliability of this measurement is questioned by many authors, ${ }^{12}$ and the value of the threshold gradient is still unclear (4 to $10 \mathrm{~mm} \mathrm{Hg}$ ). It has been demonstrated that some patient-related parameters, such as general anesthesia or recent therapeutic CSF drainage, may significantly modify the intracranial venous pressures. , $7,12^{2}$ Otherwise, some technical limitations of both manometric and ComboWire measurements should be considered in the exploration of intracranial 

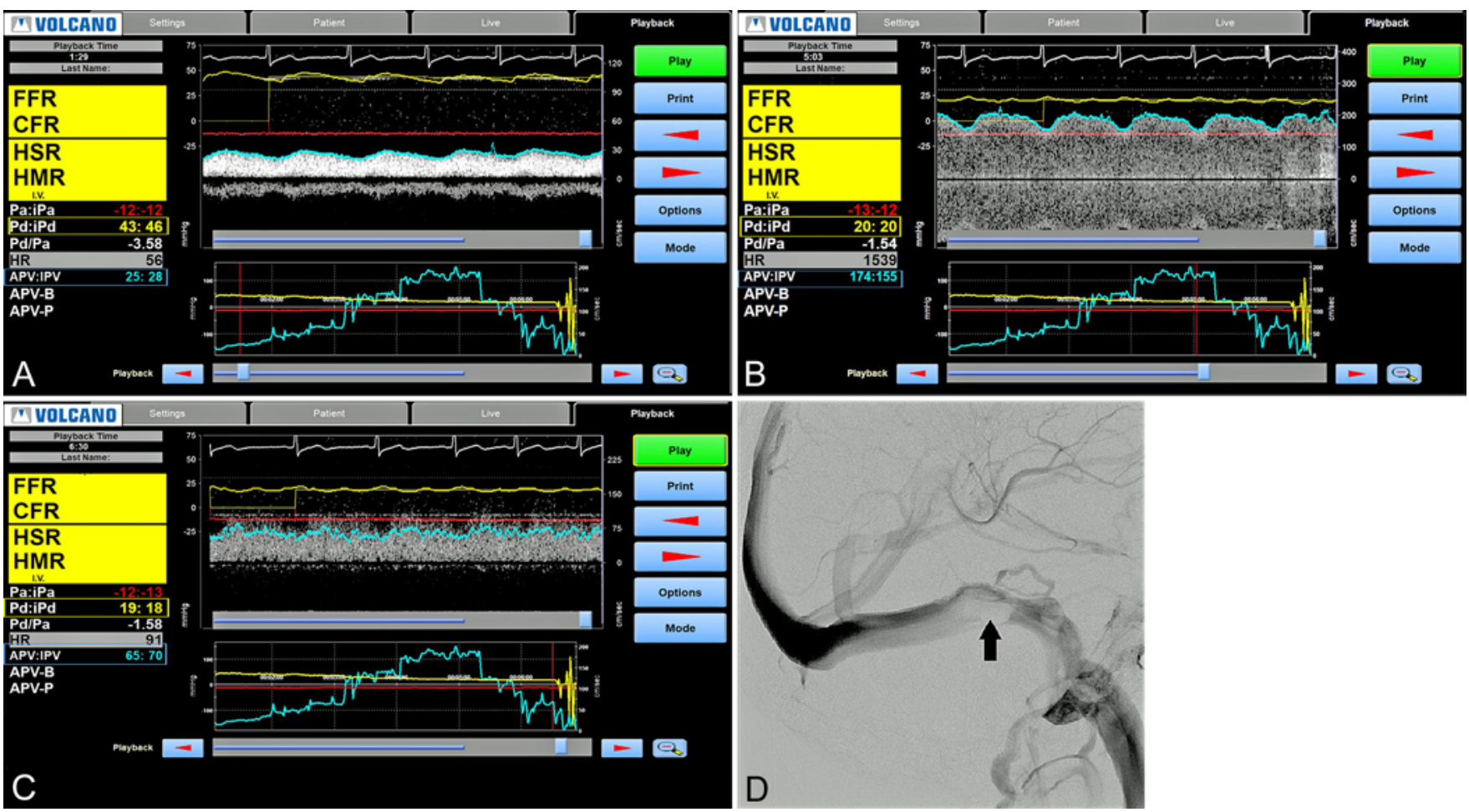

FIG. 2. Angiographic and hemodynamic exploration of a 46-year-old patient with a right ear PT associated with a right lateral sinus stenosis caused by an intraluminal arachnoid granulation (see arrow in D). We noted a significant acceleration of the BFV inside the stenosis (B, APV: $174 \mathrm{~cm} / \mathrm{s})$ compared to above $(A, 25 \mathrm{~cm} / \mathrm{s})$ and below $(C, 65 \mathrm{~cm} / \mathrm{s})$ the stenosis, and a transstenotic gradient of $24 \mathrm{~mm} \mathrm{Hg}$ ( $A, P d=43 \mathrm{~mm} \mathrm{Hg}$ in the superior sagittal sinus; $C, P d=19 \mathrm{~mm} \mathrm{Hg}$ in the sigmoid sinus). A: Measurement of venous pressures (Pd, yellow) and BFVs (APV) in the superior sagittal sinus upstream of the stenosis. B: Measurement of venous pressures (Pd, yellow) and BFVs (APV) into the stenosis. C: Measurement of venous pressures (Pd, yellow) and BFVs (APV) in the sigmoid sinus downstream of the stenosis. D: Venous phase of the conventional angiogram showing the endoluminal granulation (arrow). Note that the right lateral sinus is dominant. APV = average peak velocity; $\mathrm{Pd}=$ distal pressure (measured with the ComboWire). Figure is available in color online only.

venous pressures. We used a Prowler Select Plus microcatheter for the manometric measurement of the venous pressures in our series. Avery et al. have tested the precision of such a microcatheter in manometric pressure measurement in a flow model of venous sinus stenoses, and they found an error range of $\pm 2 \mathrm{~mm} \mathrm{Hg}$ (range 0-20 $\mathrm{mm}$ $\mathrm{Hg}) .{ }^{1}$ They demonstrated that venous pressures measured with neurovascular microcatheter were lower than pressures measured with a high-fidelity microcatheter. ${ }^{1}$ On the other hand, most experiences with the ComboWire come from the cardiology literature, where studies have documented that $<10 \%$ of such measurements fell outside the acceptable range of $\pm 2 \mathrm{~mm} \mathrm{Hg},{ }^{3}$ while the error range of the ComboWire given by the manufacturer is $\pm 3 \mathrm{~mm} \mathrm{Hg}$ (range -30 to $+100 \mathrm{~mm} \mathrm{Hg}$ ). Regarding this error range of at least $\pm 2 \mathrm{~mm} \mathrm{Hg}$ for both systems, it seems technically hazardous to propose low-threshold gradients such as 4 $\mathrm{mm} \mathrm{Hg}$ to assess the indication for stenting in patients with dural-sinus stenoses, even if the use of a ratio rather than a fixed empirical figure minimizes any potential error introduced from transducer error drift. Thus, the measurement of the blood flow velocity appears to be an interesting complementary tool for the exploration of venous pressures in patients with low pressure gradients $(<10 \mathrm{~mm}$
$\mathrm{Hg}$ ) and a clinical picture suggestive of a symptomatic LS stenosis. Seven patients in our series had a TSG lower than $10 \mathrm{~mm} \mathrm{Hg}$, but all of them had a significant increased BFV in the stenosis (i.e., a ratio [velocity in the superior sagittal sinus/velocity in the stenosis] $<0.5$ ). The PT disappeared and the venous BFVs were normalized after stent placement in all patients. This suggests that the reliability of the TSG pressure measurement alone was not sufficient to assess the indication for stenting in half of the patients with PT, while the sensitivity of the BFV appeared to be better.

In comparison to the manometric measurement, which provides measurement of only one variable, the ComboWire provides accurate flow and pressure measurements (Fig. 2). The ComboWire was first developed for advanced physiological assessment of fractional and coronary flow reserves in cases of coronary artery stenosis. ${ }^{9}$ It was used in the neurovascular area in small case studies to assess the hemodynamic consequences of flow diversion for intracranial aneurysms $\mathrm{s}^{8,13}$ or of partial embolization of brain arteriovenous malformations. ${ }^{10}$ However, its use in brain arterial structures is not in widespread clinical practice at present because of the risk of perforation and of thrombus formation, on one hand, and of the absence of clear clinical benefit resulting for such hemo- 
dynamic measurements, on the other hand. Navigation of the ComboWire in the dural sinuses appears to be safer than navigation in the cerebral arteries. The risk of perforation is very low since the dural sinuses are covered by the dura mater, and the occurrence of a thrombotic complication is unlikely in the venous circulation. We found that the VBF was significantly accelerated inside the stenosis. This acceleration, as well as the PT, resolved in all patients following stent placement. This finding supports the pathophysiological hypothesis suggesting that the tinnitus is caused by the acceleration and turbulence of the blood flow in the LS stenosis, close to the inner ear. ${ }^{2}$ The acceleration of the BFV in the stenosis therefore seems to be a more reliable hemodynamic criterion than the measurement of the TSG alone to underscore the role of the stenosis in the development of the bruit. The capability of a dual-sensor wire to simultaneously obtain intravenous pressure and BFV appears to be especially promising in this clinical scenario.

\section{Limitations of the Study}

The small number of patients is a main limitation of this study; however, this was a hypothesis-generating observational study, and we plan on further following such patients going forward.

Also, the lack of a comparative group is another limitation; however, the clinical and radiological assessments of patients presenting with PT in our institution are quite thorough, and we have previously published our results in this area. ${ }^{6}$ In our previous series, only 1 of 14 patients had persistent tinnitus after stent placement. In the present observational study, in patients with isolated PT, stenting was indicated on clinical and radiological grounds, not based on hemodynamic parameters. In patient with IIH, a threshold TSG of $>4 \mathrm{~mm} \mathrm{Hg}$ was used to assess the indication for stenting.

Finally, in 2 patients, the measurements with the ComboWire could not be performed after stenting because of a dysfunction of the wire at the end of the procedure.

\section{Conclusions}

The use of a dual-sensor guidewire to measure both pressures and velocities across a stenotic lesion of the venous sinus seems to be an effective diagnostic strategy that can help assess the indications for stenting. This approach may prove superior to the measurement of pressures alone, especially in patients with low TSG $(<10 \mathrm{~mm}$ $\mathrm{Hg}$ ) for which technical biases cannot be avoided. This combined approach for hemodynamic assessment of venous dural sinus stenoses with both venous pressures and BFVs warrants further investigation and larger comparative studies.

\section{References}

1. Avery MB, Sambrano S, Khader Eliyas J, Eesa M, Mitha AP: Accuracy and precision of venous pressure measurements of endovascular microcatheters in the setting of dural venous sinus stenosis. J Neurointerv Surg [epub ahead of print], 2017

2. Baomin L, Yongbing S, Xiangyu C: Angioplasty and stenting for intractable pulsatile tinnitus caused by dural venous sinus stenosis: a case series report. Otol Neurotol 35:366-370, 2014

3. Jeremias A, Maehara A, Généreux P, Asrress KN, Berry C, De Bruyne B, et al: Multicenter core laboratory comparison of the instantaneous wave-free ratio and resting $\mathrm{Pd} / \mathrm{Pa}$ with fractional flow reserve: the RESOLVE study. J Am Coll Cardiol 63:1253-1261, 2014

4. Kumpe DA, Seinfeld J, Huang X, Mei Q, Case DE, Roark $\mathrm{CD}$, et al: Dural sinus stenting for idiopathic intracranial hypertension: factors associated with hemodynamic failure and management with extended stenting. J Neurointerv Surg 9:867-874, 2017

5. Leach JL, Jones BV, Tomsick TA, Stewart CA, Balko MG: Normal appearance of arachnoid granulations on contrastenhanced CT and MR of the brain: differentiation from dural sinus disease. AJNR Am J Neuroradiol 17:1523-1532, 1996

6. Lenck S, Labeyrie MA, Vallee F, Saint-Maurice JP, Guillonnet A, Bernat AL, et al: Stent placement for disabling pulsatile tinnitus caused by a lateral sinus stenosis: a retrospective study. Oper Neurosurg (Hagerstown) 13:560-565, 2017

7. Lenck S, Vallée F, Labeyrie MA, Touitou V, Saint-Maurice JP, Guillonnet A, et al: Stenting of the lateral sinus in idiopathic intracranial hypertension according to the type of stenosis. Neurosurgery 80:393-400, 2017

8. Levitt MR, McGah PM, Aliseda A, Mourad PD, Nerva JD, Vaidya SS, et al: Cerebral aneurysms treated with flowdiverting stents: computational models with intravascular blood flow measurements. AJNR Am J Neuroradiol 35:143-148, 2014

9. Melikian N, Del Furia F, Di Mario C: Physiologic lesion assessment during percutaneous coronary intervention. Cardiol Clin 28:31-54, 2010

10. Orlov K, Panarin V, Krivoshapkin A, Kislitsin D, Berestov V, Shayakhmetov T, et al: Assessment of periprocedural hemodynamic changes in arteriovenous malformation vessels by endovascular dual-sensor guidewire. Interv Neuroradiol 21:101-107, 2015

11. Puffer RC, Mustafa W, Lanzino G: Venous sinus stenting for idiopathic intracranial hypertension: a review of the literature. J Neurointerv Surg 5:483-486, 2013

12. Satti SR, Leishangthem L, Chaudry MI: Meta-analysis of CSF diversion procedures and dural venous sinus stenting in the setting of medically refractory idiopathic intracranial hypertension. AJNR Am J Neuroradiol 36:1899-1904, 2015

13. Schneiders JJ, VanBavel E, Majoie CB, Ferns SP, van den Berg R: A flow-diverting stent is not a pressure-diverting stent. AJNR Am J Neuroradiol 34:E1-E4, 2013

\section{Disclosures}

The dual-sensor guidewires (ComboWire) were provided by Volcano Corporation (Philips).

\section{Author Contributions}

Conception and design: Lenck, Vallée. Acquisition of data: Lenck, Vallée, Saint-Maurice, Hong. Analysis and interpretation of data: Lenck, Vallée. Drafting the article: Vallée. Critically revising the article: Vallée, Civelli, Saint-Maurice, Nicholson, Hong, Houdart. Reviewed submitted version of manuscript: all authors. Approved the final version of the manuscript on behalf of all authors: Lenck. Statistical analysis: Lenck. Study supervision: Vallée, Houdart.

\section{Correspondence}

Stéphanie Lenck: Hôpital Lariboisière, Paris, France. stephanie. lenck@gmail.com. 